\title{
ANÁLISIS CONTRASTIVO DE LAS CARACTERÍSTICAS SEMÁNTICAS DE LAS PREPOSICIONES MONOLÉCTICAS DEL ESPAÑOL Y DEL GRIEGO MODERNO
}

\author{
CONTRASTIVE ANALYSIS OF THE SEMANTIC FEATURES OF SPANISH AND \\ MODERN GREEK MONOLECTICAL PREPOSITIONS
}

\author{
IsAaC Gómez LAGuna \\ Universidad Aristóteles de Salónica \\ isaacgomezlaguna@yahoo.com
}

Enviado: $18 / 02 / 2018$

Aceptado: 08/05/2018

\section{Resumen}

En el presente artículo contrastamos las preposiciones monolécticas del español y del griego moderno mediante el análisis componencial, enfoque semántico que considera que el significado de todo elemento lingüístico es único y permanente, no depende de factores extralingüísticos, está compuesto por semas (rasgos de significado mínimos) e interactúa con los otros significados de su contexto dando paso a los sentidos: realizaciones semánticas concretas que, a su vez, posibilitan la interpretación de determinadas estructuras funcionales. Con esta investigación queremos poner de manifiesto que: (1) el análisis componencial permite comparar elementos de distintas lenguas de la misma manera que compara elementos de una misma lengua, contrastando sus semas; (2) cuanto más similares sean los semas de dos preposiciones

\begin{abstract}
In the present article, we contrast the monolectical prepositions of the Spanish and Modern Greek language using the componential analysis. This semantic method postulates that the meaning of each linguistic element is unique and permanent, it does not depend on extralinguistic factors and it is composed by semes (basic meaning features). This meaning interacts with the other meanings of the same linguistic environment, developing senses; concrete semantic materialization which, in their turn, allow the interpretation of certain functional structures. With this research we intend to demonstrate the following: (1) the componential analysis allow us to compare elements from different languages in the same way that it compares elements of the same language, by contrasting their semes; (2) the more semes
\end{abstract}

Para citar este artículo / To cite this article: Gómez Laguna, Isaac (2018). Análisis contrastivo de las características semánticas de las preposiciones monolécticas del español y del griego moderno. ELUA, 32: 155-177. doi: 10.14198/ELUA2018.32.7

Enlace / Link: http://dx.doi.org/10.14198/ELUA2018.32.7 
de lenguas diferentes, tantas más posibilidades tienen estas preposiciones de aparecer en contextos interlingüísticamente equivalentes desarrollando sentidos contextuales semejantes; (3) la norma lingüística de una lengua restringe o potencia los diferentes usos de cada elemento lingüístico, pero no puede crear sentidos no justificados por los semas de esos elementos.

PALABRAS CLAVE: semántica, preposiciones, análisis contrastivo (español-griego moderno), análisis componencial. two prepositions from different languages share, the more likely these prepositions are to appear in inter-linguistically equivalent contexts and to develop similar contextual senses; (3) the linguistic norm of a language restricts or potentiates the different uses of each linguistic element, however it cannot create any senses which are unjustified by its semes.

KEYWORDS: semantics, prepositions, contrastive analysis (Spanish-Modern Greek), componential analysis.

\section{LOS PARADIGMAS PREPOSICIONALES DEL ESPAÑOL Y DEL GRIEGO}

No pretendemos esclarecer cuáles son los elementos que conforman los paradigmas preposicionales del español y del griego moderno (a partir de ahora, simplemente griego), pues se trata de un asunto especialmente polémico (Trujillo Carreño 1971: 238; Theophanopoúlou-Kontoú 1995: 233-235; Fykias 1997: 439-440; Laguna Campos 2005: 526), pero debemos necesariamente determinar cuáles son los elementos objeto de nuestro análisis.

Para ello hemos tomado como base los cuarenta y cuatro elementos griegos y los cincuenta españoles a los que se ha atribuido naturaleza preposicional en las gramáticas de las últimas décadas (Gómez Laguna 2014: 72, 98), y hemos excluido aquellos elementos que: (1) No tienen libertad sintáctica o la tienen únicamente en niveles muy específicos de la lengua; (2) Adoptan función preposicional únicamente bajo condiciones específicas; (3) Comparten más características con otros paradigmas que con el preposicional.

Dadas las naturales limitaciones de espacio en artículos como el presente, nos resultaría imposible indicar y ejemplificar las razones que nos han llevado a excluir de nuestro análisis un buen número de elementos griegos y españoles a los que en algún momento se les ha atribuido naturaleza preposicional ${ }^{1}$. Así, sin negar a priori las objeciones que se puedan presentar ante la adopción de las preposiciones seleccionadas, y sin afirmar que éstas sean necesariamente las únicas que conforman los paradigmas preposicionales del español y del griego, hemos considerado que las preposiciones objeto de nuestro estudio son las siguientes ${ }^{2}$ :

\footnotetext{
1 La lista total de elementos a los que en alguna ocasión se les ha atribuido naturaleza preposicional en español es: A, AD, ADONDE, ALlENDE, ANTE, APUD, AQUENDE, BAJO, CABE, CABO, CARA, COMO, CON, CONTRA, CUANDO, DE, DEJANTE, DENDE, DES, DESDE, DONDE, DURANTE, EN, ENTA, ENTRE, ENTRO, ESCONTRA, EXCEPTO, EXTRA, FASTA, HACIA, HASTA, IN, INCLUSO, MEDIANTE, MENOS, PAR, PARA, POR, PORA, PRO, SALVO, SEGÚN, SEN, Sin, SO, SOBRe, TRAS, VERSUS y VÍA (Seco Reymundo 1996; De Bruyne 1999; Morera Pérez 1999; Marcos Marín \& España Ramírez 2001; Lamíquiz Ibáñez 2001; Alarcos Llorach 2001; RAE 2001 y 2009; Gómez Laguna 2014).

Por su parte, los elementos griegos que en algún momento han sido considerados preposiciones son: ANÁ, ANÉU, ANTí, APÓ, GIA, DÍCHOS, DÍA, EIS, EK/EX, EKTÓS, EN, ÉNANTI, ENANTÍON, ÉNEKA, ENTÓS, ENÓPION, EXAITÍAS, EPÍ, EÓS, ÍSAME, KATÁ, KETÓPIN, LÓGO, ME, MEÍON, MÉSO, METÁ, METAXÝ, MÉCHRI, PARÁ, PAROUSÍA, PERÍ, PLIN, PRIN, PRO, PROS, SAN, SE, SYN, YPÉR, YPÓ, CHÁRIN, CHORÍs y OS (Thomópoulos 1945; Triandafyllídis 1978; Bampiniótis 2006; Chatzisavvidis 2009; Klaíris y Bampiniótis 2009; Holton et al. 2009; Bortone 2010; Gómez Laguna 2014).

2 Para la transliteración de caracteres griegos seguimos las normas de ONU, ELOT 743/1987.
} 
- Español: A, ANTe, bajo, Con, CONTRA, De, Desde, en, entre, hacia, hasta, para, por, SEGÚN, SIN, SOBRE Y TRAS.

- Griego ${ }^{3}$ : ANTí, APÓ, GiA, KATÁ (con acusativo ${ }^{4}$ ), ME, METAXÝ, MÉChri (y su variante Éos), PARÁ, PROS, SE Y CHORÍS (y su variante DíchOS).

\section{CARACTERÍSTICAS DE LAS PREPOSICIONES DEL ESPAÑOL Y DEL GRIEGO}

La semejanza estructural del griego y del español (Petroúnias 1996: 37-40; Leontaridi 2008 [2001]: 7-9; 2011: 93-113) permite establecer las características de las preposiciones de estas lenguas conjuntamente desde planteamientos morfológicos, fonológicos, semánticos y sintácticos, así como atendiendo a su naturaleza léxica y gramatical:

a. Morfología. Las preposiciones monolécticas constan de una única pieza léxica sin flexión gramatical.

b. Fonología. Las preposiciones tienden a la atonicidad: en griego son átonas las denominadas preposiciones básicas, APÓ, GIA, ME y SE, mientras que en español lo son todas, excepto SEGún. Tanto es así, que en ambas lenguas los elementos de otros paradigmas usados con función preposicional suelen perder su tonicidad (Setátos 1995: 872-873; Morera Pérez 1999: 105).

c. Semántica. El significado de cada preposición es único y está compuesto, como veremos, por rasgos de significado denominados semas. No obstante, este significado no se manifiesta por sí mismo en contextos concretos, sino que desarrolla diversos sentidos contextuales, fruto de la interacción semántica entre la propia preposición, el regente (elemento del que la preposición depende) y el término (elemento que la preposición introduce), pues la preposición subordina semánticamente éste a aquel, y forma junto con el término la unidad semántica y funcional denominada grupo preposicional (Thomópoulos 1945: 79; Pottier 1970: 101; Trujillo Carreño 1988: 37; Lamíquiz Ibáñez 2001: 183; Clairis-Babiniotis 2009: 916; RAE 2009: 2223). Es imposible indicar de modo general qué es lo que cada uno de estos elementos aporta para el desarrollo de los sentidos, pues ello depende de las características semánticas de cada preposición, regente y término, por lo que debe ser analizado en cada contexto particular. Sin embargo, se ha de tener en cuenta que estas características serán las que permitan o impidan la aparición de una u otra preposición, $\mathrm{y}$, en caso de que aparezca, los sentidos contextuales que pueda desarrollar.

d. Sintaxis. Cada sentido semántico contextual ('causa', 'medio', 'tiempo', etc.) se actualiza como una determinada función sintáctica, de lo que se desprende que la función semántica relacionante y subordinante de las preposiciones es también sintáctica (Osuna García 1991: 106; Cifuentes Honrubia 1998: 113).

3 Quizá los elementos más polémicos sean PRIN y METÁ. Sin embargo, sin tomar una posición absoluta acerca de ello, no los incluimos en nuestro análisis porque se asemejan a los adverbios en que pueden aparecer tanto sin complemento como complementados por grupos preposicionales introducidos por APÓ.

4 Es diferente el adverbio KATÁ, que puede funcionar con autonomía sintáctica o complementado por genitivo, y es siempre tónico (Gómez Laguna 2014: 13). No obstante, el simple hecho de que el término esté en genitivo no basta para explicar que exista un KATÁ adverbial, pues hay preposiciones como METAXÝ o ANTí que encabezan términos en genitivo. Por otro lado, MÉCHRI conserva, junto a la construcción con acusativo, usos arcaizantes con término en genitivo sin que ello conlleve un cambio categorial. 
e. Naturaleza. Entendiendo que la naturaleza léxica es aquella que se refiere a la realidad extralingüística, y la naturaleza gramatical es la que afecta a los elementos de un mismo enunciado (Pavlídou, 1992: 107), las preposiciones presentan características mixtas:

- Características léxicas. Aunque sólo algunas preposiciones como ANTE, BAJO, SOBRE y TRAS presentan significado léxico (RAE 2009: 2225), todas las preposiciones seleccionan semánticamente a sus términos, tal y como hacen las categorías léxicas (Horno Chéliz 2002: 117-118, 171). Así, el rasgo ‘extensión’ de EN (que veremos más adelante) permite seleccionar un elemento durativo como verano, pero no uno puntual como tres:

(1) Nos veremos en verano/*en las tres 5

- Características gramaticales. Las principales características gramaticales de las preposiciones son: (1) No se relacionan directamente con la realidad extralingüística, sino únicamente a través de su término (Pavlídou 1992: 107; Osuna 2008: 627-628); (2) Seleccionan gramaticalmente a su término ${ }^{6}$, pues exigen que éste tenga, inherente o contextualmente, rasgos nominales ${ }^{7}$. Tienen, además, otras características que las acercan a los elementos gramaticales, como: poca libertad sintáctica; significado poco específico; gran frecuencia de uso; poca fuerza tónica; no constituyen sintagmas por sí mismas; conforman un paradigma cerrado; y es difícil su sustitución paradigmática (Horno Chéliz 2002: 159-161, 171-174).

Llegados a este punto, hemos de puntualizar que el proceso histórico mediante el que se conformó el griego actual, hace que algunas de sus preposiciones presenten particularidades. Simplificando mucho se puede afirmar que hasta el siglo XIX convivieron dos variantes del griego, el clásico, mantenido convencionalmente en la educación y en los entornos oficiales, y el demótico, la lengua hablada por el pueblo evolucionada de manera natural. Para resolver esta dicotomía, en el siglo XIX se implantó artificialmente el katharevousa, variante lingüística que trajo consigo muchos elementos sintácticos, morfológicos y léxicos del griego clásico ${ }^{8}$. Entre estos elementos, había un buen número de preposiciones que tuvieron mejor o peor fortuna en su implantación final en el griego actual. Muchas dejaron de usarse y otras quedaron restringidas a expresiones fosilizadas, pero algunas continúan siendo empleadas de manera productiva hoy en día (Bortone 2010: 292); aunque ocasionalmente presentan restricciones sintácticas y semánticas que mencionaremos cuando corresponda. Por otro

5 Salvo que se indique otro origen, los ejemplos griegos y españoles propuestos para ser contrastados, así como las traducciones al español de algunos ejemplos griegos que no tienen correspondencia en español, son obra nuestra. 6 Horno Chéliz $(2002$ : 117-118, 171) considera que las preposiciones no hacen selección gramatical, lo que la lleva a postular que tienen naturaleza léxica.

7 Los casos en los que los adjetivos funcionan como término no son usos libres, sino lexicalizaciones y fijaciones (Trujillo Carreño 1971: 247-248; Morera Pérez 1988: 49).

Pueden ser término de preposición: (1) sustantivos; (2) adverbios nominales; (3) oraciones sustantivadas; (4) oraciones de relativo sin antecedente expreso, porque contienen la noción de un antecedente sustantivo; (5) grupos preposicionales que cumplen con la función referencial prototípicamente nominal; y (6) cualquier sintagma que presente contextualmente rasgos nominales (Pavón Lucero 1999: 605; Holton et alii 1999: 422-424, RAE 2009: 51, 1565, 2226, 3332, 3335; Klaíris y Bampiniótis 2009: 926-929).

8 Sobre la cuestión lingüística del griego, véase Bádenas de la Peña (1988: 303-33), Rodríguez Adrados (1999), Archákis y Kondýli (2002: 103-109) y Papanastassíou (2010), entre otros. 
lado, también se ha de tener en cuenta que prácticamente las únicas preposiciones empleadas en demótico eran APÓ, GIA, ME y SE; por lo que en la actualidad siguen siendo las preposiciones con mayor número de usos libres, fosilizados y gramaticalizados, y se denominan preposiciones básicas (Setátos 1995: 872-873).

\section{ENFOQUE SEMÁNTICO}

El significado de las preposiciones es lo que permite entender su capacidad combinatoria y, por lo tanto, los sentidos contextuales que los grupos preposicionales pueden desarrollar (Pottier 1970: 100; Trujillo Carreño 1975: 303-304; Cifuentes Honrubia 1998: 113). En nuestro estudio empleamos concretamente el método del análisis componencial (Lyons 1997: 129-155), enfoque monosemántico que considera que el significado de todo elemento lingüístico es una abstracción que presenta las siguientes características: (1) Es único e independiente del contexto; (2) Está formado por uno o más semas (rasgos de significado mínimos) de cuya combinación surge la definición componencial de cada palabra, es decir su significado; (3) Se diferencia de otros significados en al menos un sema; (4) Interactúa con los significados de los elementos lingüísticos presentes en un determinado contexto para desarrollar sentidos contextuales concretos, que son lo que los hablantes perciben (Goodenough 1956; Katz y Fodor 1963; Pottier 1970; Trujillo 1971; Kempson 1977; Leech 1977; Bampiniótis 1985; Morera Pérez 1988; Lyons 1997; Horno Chéliz 2002; Rodríguez Zamora 2004).

Este método es especialmente adecuado para el contraste interlingüístico de las preposiciones porque: (1) Parte de planteamientos puramente lingüísticos no extralingüísticos $\mathrm{y}$, como se ha indicado, las preposiciones no poseen referente extralingüístico directo; (2) Reduce a uno solo los múltiples significados habitualmente atribuidos a las preposiciones; (3) Contrasta elementos de distintas lenguas de la misma manera que contrasta elementos de una misma lengua, mediante la confrontación de sus semas.

\subsection{Semas de las preposiciones griegas y españolas}

Hay varios trabajos que clarifican los semas de las preposiciones españolas, entre los que destacan los de Pottier (1964; 1970), Trujillo Carreño (1971) y, muy especialmente, Morera Pérez $(1988 ; 1999)$. Sin embargo, las preposiciones griegas únicamente se han analizado desde esta perspectiva en nuestra tesis doctoral inédita (Gómez Laguna 2014), para lo que nos hemos basado en las investigaciones de los tres autores indicados y de otros como Goodenough (1956), Katz y Fodor (1963), Kempson (1977), Lyons (1997) y Rodríguez Zamora (2004).

Aunque no es objeto de este artículo explicar el proceso de identificación de los semas de las preposiciones del griego, debemos al menos indicar las pautas que hemos seguido para ello:

1. Identificar los contextos en los que aparece cada preposición.

2. Usar las pruebas de la conmutación (emplear diferentes preposiciones en un mismo contexto lingüístico) y la distribución (utilizar una misma preposición en distintos contextos).

3. Analizar las influencias semánticas recíprocas entre cada preposición y el resto de los elementos de sus entornos lingüísticos. 
4. Abstraer los rasgos comunes (semas) que se repiten en cada contexto.

5. Repetir este proceso inversamente: desde los semas hasta las preposiciones.

6. Oponer semánticamente las preposiciones del paradigma preposicional griego para identificar las diferencias semánticas que presentan y la funcionalidad del paradigma como conjunto.

No obstante, antes indicar los semas de las preposiciones griegas y españolas, y de contrastarlos entre sí, hemos de referirnos al grado en que se presentan los semas, a los semas concomitantes y a las neutralizaciones de semas:

- Grado de los semas. Las preposiciones caracterizadas por un determinado sema poseen este rasgo en grado positivo (+). Sin embargo, un sema también puede ser el correlato negativo de otro, en cuyo caso es posible emplear denominaciones diferentes con cada uno estos semas o marcar uno de ellos con el grado negativo (-); es decir, el sema opuesto a la 'extensión', puede denominarse tanto 'puntualidad' como '-extensión'. Y, por último, las preposiciones que no presentan un determinado sema son indiferentes a él; habitualmente esto no se marca de ninguna manera, pero por cuestiones metodológicas en algunos casos lo representaremos como grado neutro ( \pm ) (Lyons, 1997: 136). Veremos que esto es algo importante para establecer, por ejemplo, las relaciones entre DE ('-extensión'), DESDE ('+extensión’) y APÓ (' \pm extensión’).

f. Semas concomitantes. Son el resultado de la combinación de los semas de un determinado significado; no son sentidos contextuales porque existen siempre que se produce esa combinación de semas, con independencia del contexto, y porque interactúan con otros semas desarrollando sentidos contextuales propiamente dichos. Así, los semas de POR, 'movimiento con interioridad y extensión', conllevan el sema concomitante ‘tránsito' (Morera Pérez 1988: 287); mientras que la 'contraposición y exclusión' de la preposición griega ANTí conllevan 'sustitución' (Gómez Laguna 2014: 149-150).

g. Neutralización de semas. Algunos de los semas que componen un determinado significado no tienen la posibilidad de actualizarse en ciertos entornos, por lo que se neutralizan, permitiendo que el resto de los semas sigan interactuando con el contexto y desarrollando sentidos contextuales. Véase lo que sucede con HACIA, cuyos semas son 'movimiento de proximidad':

(2) Viene hacia el parque. 'movimiento de proximidad'

(3) Hacia el mediodía saldrá el sol. 'orientación/proximidad temporal'

(4) La casa tiene balcones hacia el mar. 'orientación espacial'

El contexto de (2) es dinámico, por lo que los semas 'movimiento de proximidad' de HACIA permanecen invariables. Por el contrario, los contextos de (3) y (4) son respectivamente temporal y espacial no dinámico, lo que hace que HACIA neutralice su 'movimiento' y desarrolle sentidos de 'proximidad temporal' y 'orientación espacial'.

Aclarado lo anterior, podemos exponer los semas que caracterizan a las preposiciones de ambos idiomas (seguimos un simple orden alfabético). 


\begin{tabular}{|c|c|c|c|}
\hline acompañamiento & & horizontal & $\rightarrow$ sólo español \\
\hline alejamiento & & interior & \\
\hline proximidad & & movimiento & \\
\hline bloqueo & $\rightarrow$ sólo español & múltiple & \\
\hline contable & $\rightarrow$ sólo griego & negativo & \\
\hline contacto & & positivo & \\
\hline contraposición & $\rightarrow$ sólo griego & referencia externa & \\
\hline determinación & & adecuación & \\
\hline exclusión & $\rightarrow$ sólo griego & ubicación & \\
\hline extensión & & vertical & $\rightarrow$ sólo español \\
\hline
\end{tabular}

Tabla 1. Semas de las preposiciones griegas y españolas

Tres semas son exclusivos de las preposiciones griegas ('contable', 'contraposición' y 'exclusión'), tres de las preposiciones españolas ('bloqueo', 'horizontal' y 'vertical') y catorce son comunes a ambos idiomas; es un alto porcentaje que justifica que, como veremos, algunas preposiciones griegas y españolas tengan combinaciones de semas muy similares o incluso idénticas.

En las dos tablas que siguen se muestran por separado tanto los semas que caracterizan a las preposiciones españolas como sus definiciones componenciales de manera analítica:

\begin{tabular}{|c|c|c|c|c|c|c|c|c|c|c|c|c|c|c|c|c|c|c|c|c|}
\hline $\begin{array}{l}\text { Grado del } \\
\text { sema: } \\
(+) \text { positivo } \\
\text { (-) negativo }\end{array}$ & 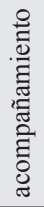 & 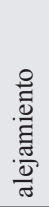 & 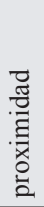 & $\begin{array}{l}\frac{8}{2} \\
\frac{0}{0}\end{array}$ & $\begin{array}{l}\frac{0}{0} \\
\frac{\pi}{0} \\
0 \\
0\end{array}$ & 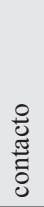 & 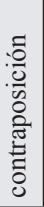 & 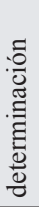 & 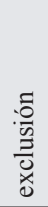 & 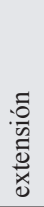 & 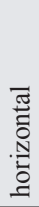 & 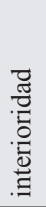 & 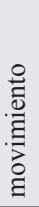 & 莺 & 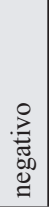 & 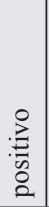 & 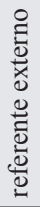 & 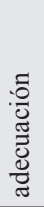 & $\begin{array}{l}: \frac{0}{0} \\
.00 \\
\frac{0}{3}\end{array}$ & 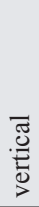 \\
\hline $\mathrm{a}$ & & & + & & & + & & & & - & & & + & & & & & & & \\
\hline ante & & & & & & & & & & & + & & & & & + & + & & + & \\
\hline bajo & & & & & & & & & & & & & & & + & & + & & + & + \\
\hline con & + & & & & & & & & & & & & & & & + & & & & \\
\hline contra & & & + & + & & & & & & & & & + & & & & & & & \\
\hline de & & + & & & & & & & & - & & & + & & & & & & & \\
\hline desde & & + & & & & & & & & + & & & + & & & & & & & \\
\hline en & & & & & & & & & & + & & + & & & & & & & + & \\
\hline entre & & & & & & & & & & & & & & + & & & + & & + & \\
\hline hacia & & & + & & & & & & & & & & + & & & & & & & \\
\hline hasta & & & + & & & + & & & & + & & & + & & & & & & & \\
\hline para & & & + & & & & & + & & & & & + & & & & & & & \\
\hline por & & & & & & & & & & + & & + & + & & & & & & & \\
\hline según & & & & & & & & & & & & & & & & & & + & & \\
\hline $\sin$ & + & & & & & & & & & & & & & & + & & & & & \\
\hline sobre & & & & & & & & & & & & & & & & + & + & & + & + \\
\hline tras & & & & & & & & & & & + & & & & + & & + & & + & \\
\hline
\end{tabular}

Tabla 2. Semas de las preposiciones españolas 


\begin{tabular}{|l|l|}
\hline a & 'movimiento de proximidad sin extensión y con contacto' \\
\hline ante & 'ubicación con referencia externa horizontal y positiva' \\
\hline bajo & 'ubicación con referencia externa horizontal y negativa' \\
\hline con & 'acompañamiento positivo' \\
\hline contra & 'movimiento de proximidad con bloqueo' \\
\hline de & 'movimiento de alejamiento sin extensión' \\
\hline desde & 'movimiento de alejamiento con extensión' \\
\hline en & 'ubicación con interioridad y extensión' \\
\hline entre & 'ubicación con referencia externa múltiple' \\
\hline hacia & 'movimiento de proximidad' \\
\hline hasta & 'movimiento de proximidad con extensión y contacto' \\
\hline para & 'movimiento de proximidad con determinación' \\
\hline por & 'movimiento interioridad y extensión' \\
\hline según & 'adecuación' \\
\hline sin & 'acompañamiento negativo' \\
\hline sobre & 'ubicación con referencia externa vertical y positiva' \\
\hline tras & 'ubicación con referencia externa horizontal y negativa'. \\
\hline
\end{tabular}

Tabla 3. Definiciones componenciales de las preposiciones españolas

En las tablas que se muestran a continuación aparecen los semas que caracterizan a las preposiciones griegas y sus definiciones componenciales:

\begin{tabular}{|c|c|c|c|c|c|c|c|c|c|c|c|c|c|c|c|c|c|c|c|c|}
\hline $\begin{array}{l}\text { Grado del } \\
\text { sema: } \\
(+) \text { positivo } \\
( \pm) \text { neutro }\end{array}$ & 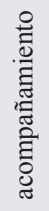 & 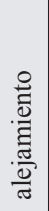 & 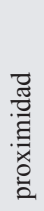 & $\begin{array}{l}8 \\
\stackrel{0}{0} \\
\frac{0}{0}\end{array}$ & $\begin{array}{l}\frac{0}{0} \\
\text { त्ञ } \\
\text { J }\end{array}$ & 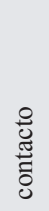 & 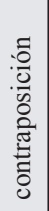 & 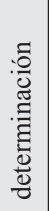 & $\frac{: 0}{0}$ & 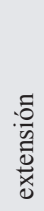 & 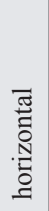 & 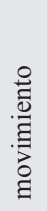 & 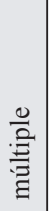 & 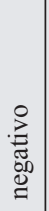 & 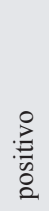 & 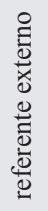 & 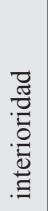 & 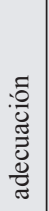 & 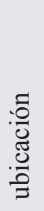 & : \\
\hline antí & & & & & & & + & & + & & & & & & & & & & & \\
\hline apó & & + & & & & & & & & \pm & & + & & & & & & & & \\
\hline gia & & & + & & & & & + & & & & + & & & & & & & & \\
\hline chorís & + & & & & & & & & & & & & & + & & & & & & \\
\hline katá & & & & & & & & & & & & & & & & & & + & & \\
\hline me & + & & & & & & & & & & & & & & + & & & & & \\
\hline méchri & & & + & & & + & & & & + & & + & & & & & & & & \\
\hline metaxý & & & & & + & & & & & & & & + & & & + & & & + & \\
\hline pará & & & & & & & + & & & & & & & & & & & & & \\
\hline pros & & & + & & & & & & & & & + & & & & & & & & \\
\hline se & & & + & & & + & & & & \pm & & + & & & & & + & & & \\
\hline
\end{tabular}

Tabla 4. Semas de las preposiciones griegas 


\begin{tabular}{|l|l|}
\hline antí & 'contraposición y exclusión' \\
\hline apó & 'movimiento de alejamiento' \\
\hline gia & 'movimiento de proximidad con determinación' \\
\hline katá & 'adecuación' \\
\hline me & 'acompañamiento positivo' \\
\hline metaxý & 'ubicación con referencia externa múltiple y contable' \\
\hline méchri & 'movimiento de proximidad con extensión y contacto' \\
\hline pará & 'contraposición' \\
\hline pros & 'movimiento de proximidad' \\
\hline se & $\begin{array}{l}\text { 'movimiento de proximidad con contacto, interioridad e } \\
\text { indiferencia a la extensión' }\end{array}$ \\
\hline chorís & 'acompañamiento negativo' \\
\hline
\end{tabular}

Tabla 5. Definiciones componenciales de las preposiciones griegas ${ }^{9}$

Cuanto mayores son las similitudes semánticas entre dos elementos lingüísticos de distintas lenguas tantas más posibilidades tienen estos elementos de aparecer en contextos interlingüísticamente semejantes y, por lo tanto, de desarrollar sentidos contextuales similares. No hay duda de que el campo de usos de una palabra depende de la norma de su propia lengua (Trujillo Carreño 1988: 307), la cual está sometida a factores lingüísticos, sin duda, pero también a factores sociales e históricos. No obstante, las similitudes semánticas entre dos elementos de lenguas diferentes facilitan y explican que estos elementos aparezcan en contextos semejantes desarrollando sentidos equivalentes. Y eso es, precisamente, lo que exponemos a continuación.

\subsection{Contraste semántico entre las preposiciones griegas y españolas}

\subsubsection{Preposiciones griegas y españolas con semas idénticos}

\subsubsection{Hasta y méchri: 'movimiento de proximidad con extensión y contacto“}

HASTA y MÉCHRI mantienen una gran uniformidad en sus sentidos contextuales porque tienen un significado idéntico y muy concreto (parafraseable como 'movimiento de proximidad que subraya la extensión del movimiento y establece contacto con el término') que no está contaminado por influencias sociales o históricas (Morera Pérez 1988: 219; Horno Chéliz 2002: 245).

(5) a. El autobús llega hasta la escuela.

b. To leoforeío ftánei méchri to scholeío.

(6) a. Estaremos aquí hasta las tres.

b. Tha eímaste edó méchri tis treis.

9 En el caso de que debiéramos ofrecer la definición componencial de PRIN y METÁ, éstas serían: PRIN, 'ubicación con referente externo único horizontal y positiva'; METÁ, 'ubicación con referente externo único horizontal y negativa'. 
La similitud semántica de HASTA y MÉCHRI se mantiene incluso cuando adoptan función adverbial focal con el valor semántico de 'incluso'; algo que sólo es justificable retrotrayéndonos a su semántica, con independencia de la categoría gramatical que en cada caso se les atribuya:

(7) a. Hasta tú robaste.

b. Méchri kai esý éklepses.

\subsubsection{Con y me: 'acompañamiento positivo' ('presencia')}

CON y ME expresan 'presencia' y, en general, aparecen en los mismos contextos desarrollando sentidos similares:

(8) a. Está con Ana.

b. Eínai me tin Ánna.

(9) a. Estoy con amigos.

b. Eímai me fílous.

Son, sin embargo, preposiciones muy antiguas (CON $<$ del latín CUM; ME $<$ del griego antiguo METÁ), por lo que han desarrollado numerosos usos gramaticales y fijaciones que no tienen correlato en la otra lengua:

(10) a. Enamorado de ti.

b. Erotevménos me eséna.

(11) a. Amigo de Nikos.

b. Fílos me ton Níko. / Fílos tou Níkou (genitivo).

En (10)b y (11)b ME está gramaticalizada y se limita a expresar una relación entre regente y término, mientras que en (11)a y (11)a esta misma relación la establece DE, el recurso español más natural para marcar relación; de hecho, en (11)b ME alterna con el genitivo, el correspondiente medio gramatical griego para expresar relación. Ahora bien, que no haya correlato en la otra lengua no supone que la fijación gramatical no tenga justificación semántica; al contrario, es precisamente la semántica de cada elemento lo que permite que una de sus características se especialice para expresar un determinado valor gramatical. Así, ME es susceptible de aparecer gramaticalizada en (10)b y (11)b precisamente porque su 'acompañamiento' expresa que la noción del regente se produce 'en compañía' física o nocional del término.

\subsubsection{Sin y CHORís: ‘acompañamiento negativo' ('ausencia')}

SIN y CHORÍs suelen aparecen en contextos similares expresando sentidos muy cercanos a su significado básico porque su baja frecuencia de empleo ha impedido que desarrollen usos gramaticalizados (Klaíris y Bampiniótis 2009: 918; Morera Pérez 1988: 437):

(12) a. Sin amigos.

b. Chorís fílous. 
(13) a. Sin decir nada.

b. Chorís na pei típota.

\subsubsection{Para y gia: 'movimiento de proximidad con determinación'}

En contextos espaciales dinámicos, estas preposiciones expresan un 'movimiento determinado a alcanzar al término'; mientras que en el resto de los contextos se neutraliza su 'movimiento', lo que hace que pasen a expresar una 'determinación' ('intencionalidad', 'objetivo'):

(14) a. Va para Creta.

b. Pigaínei gia tin Kríti.

(15) a. Tengo algo para ti.

b. Écho káti gia eséna.

No obstante, la antigüedad de PARA y GIA ha hecho que se carguen de múltiples usos que no tienen correspondencia en la otra lengua. Así, a diferencia de GIA, PARA puede neutralizar su 'determinación' y expresar 'orientación' cuando ésta se deriva del propio contexto (Morera Pérez 1988: 240; Portilla Chaves 2011: 235-237):

(16) a. La isla está para el sur.

b. To nisí eínai * gia to nóto.

(17) a. Los balcones dan para el sur.

b. Ta balkónia koitáne * gia to nóto.

GIA, por su parte, aparece en múltiples expresiones fosilizadas y, a menudo, se emplea desemantizada como simple elemento de enlace:

(18) a. Estuvimos hablando *para fútbol.

b. Milísame gia podósfairo.

(19) a. El Ministro *para el Orden Público.

b. O Ypourgós gia ti Dimósia Táxi.

\subsubsection{Hacia y pros: 'movimiento de proximidad"}

En contextos de movimiento, HACIA y PROS expresan un 'movimiento de proximidad', pero en contextos no dinámicos se neutraliza el 'movimiento', de modo que la 'proximidad' se materializa como una 'orientación' espacial o temporal:

(20) a. Va hacia casa.

b. Pigaínei pros to spíti.

(21) a. Da hacia el monte.

b. Koitáei pros to vounó.

(22) a. Vendrá hacia el mediodía.

b. Tha érthei pros to mesiméri.

HACIA tiene un significado bastante específico y una relativa juventud, lo que impide que abunde en usos fijados (Morera Pérez 1988: 225). PRos, por su parte, es una de las prepo- 
siciones reintroducidas en el griego moderno y conserva algunos de los usos que poseía en griego antiguo. Por esa razón, puede aparecer en ciertos contextos semifijados de carácter formulario-administrativo que expresan un 'objetivo' y que, en español, están reservados a PARA; cuyo significado está caracterizado por la 'determinación' ('objetivo'). Ahora bien, en griego se suele seleccionar GIA en estos mismos contextos cuando el mensaje no posee carácter formulario, pues también está caracterizada por la 'determinación':

(23) a. Pros/gia ton kýrio Níko.

b. *Hacia/para el señor Nikos.

(24) a. Pros/gia tous endiaferómenous.

b. *Hacia/para los interesados.

(25) a. Pros, gia anakoínosi.

b. *Hacia/para notificación

\subsubsection{Según y katá: ‘adecuación’10}

En sema 'adecuación' de estas preposiciones expresa una 'conformidad' del regente con respecto al término, al que interpreta como 'ámbito de referencia con adecuación al que se desarrolla la noción del regente'. Por lo tanto, estas preposiciones expresan una relación nocional entre el regente y el término, estando el primero condicionado por la designación del segundo:

(26) a. Según lo dicho.

b. Katá ta legómena.

(27) a. Según la ley.

b. Katá ton nómo.

(28) a. Según Juan.

b. Katá ton Gioánni.

La norma del español ha limitado SEGÚN a entornos nocionales en los que el término expresa preceptos, reglas u opiniones, pero la norma griega es mucho menos restrictiva, lo que permite que KATÁ aparezca también en otros contextos:

(29) Kárfosan tis pinézes katá ton áxona.

'Clavaron las chinchetas conforme al eje'.

(30) I Souidía vrísketai katá ton Vorrá.

'Suecia se encuentra hacia/por el Norte'.

(31) Ta paráthira eínai prosanatolisména katá ton Vorrá.

'Las ventanas están orientadas a/hacia el norte'.

(32) Oi nomádes anavaínoun katá ton Vorrá.

'Los nómadas suben a/hacia/para el norte'.

(33) Katá ton Emfýlio Pólemo éfygan polloí geítones.

'Durante la Guerra Civil se marcharon muchos vecinos'.

10 Marcial Morera Pérez puntualizó en el VIII Congreso Internacional de Lingüística Hispánica de la Universidad de Leipzig 26-30/09/2016, que el significado de SEGÚN es un 'seguimiento en pos del término' del que se deriva una ‘adecuación' a éste. Aunque no dudamos que el origen de SEGúN (<SECUNDUM) justifica esta propuesta, consideramos que sincrónicamente esta preposición expresa principalmente 'adecuación'. 
En todos estos casos se expresa una conformidad del regente con respecto al término. Con katá ton áxona se indica que la acción del regente se realiza 'con adecuación a la forma del eje'; en todos los ejemplos en los que aparece katá ton Vorrá se indica que la noción expresada por el verbo se desarrolla 'con adecuación/conforme al Norte', que contextualmente se interpretará como 'orientación' o 'proximidad', según sea el verbo estático o de movimiento; en katá ton Emfýlio Pólemo, el término hace referencia a un suceso dentro de unos límites temporales (la Guerra Civil), y es ello precisamente lo que dota al contexto de un carácter temporal. No es de extrañar, por lo tanto, que KATÁ pueda compartir algunos contextos con PROS, aunque por distintas razones semánticas.

\subsubsection{Preposiciones griegas y españolas con algunos semas comunes}

3.2.2.1. De: 'movimiento de alejamiento sin extensión' / Desde: 'movimiento de alejamiento con extensión' / Apó: 'movimiento de alejamiento indiferente a la extensión'

Estas preposiciones expresan un 'movimiento de alejamiento' pero se diferencian por el grado del sema 'extensión': DESDE presenta '+extensión', por lo que enfatiza el desarrollo del movimiento; DE presenta '-extensión' ('puntualidad'), por lo que se centra en el punto de origen; mientras que APÓ presenta ‘ \pm extensión’, grado neutro que no resalta ninguno de estos aspectos:

(34) a. Se extiende desde/*de Madrid.

b. Epekteínetai apó tin Madríti.

(35) a. Viene de/desde Madrid.

b. Érchetai apó ti Madríti.

El verbo EXTENDER de (34)a posee '+extensión', por lo que acepta DESDE y rechaza la 'puntualidad' de DE. En (34)b el verbo EPEKTEÍNO también está caracterizado por '+extensión', pero acepta APÓ porque esta preposición es indiferente a la 'extensión', lo que le permite aparecer en contextos puntuales y extensivos.

El verbo VENIR de (35)a es indiferente a la 'extensión', así que acepta tanto DE como DESDE; aunque la primera sólo indica el origen del movimiento, mientras que la segunda enfatiza su recorrido. Por su parte, ÉRCHOMAI en (35)b es también indiferente a la 'extensión' y, consecuentemente, acepta APÓ; no obstante, el hablante que quiera enfatizar el recorrido del movimiento tendrá que recurrir a medios léxicos:

(36) Érchetai ólo to drómo apó ti Madríti.

'Viene todo el camino desde Madrid'.

3.2.2. Entre: 'ubicación con referencia externa múltiple' / Metaxý: 'ubicación con referencia externa múltiple y contable’

Estas preposiciones ubican la noción del regente en una zona localizada entre varios puntos externos al propio término ('referencia múltiple y externa'), pero METAXÝ, a diferencia de ENTRE, está caracterizada por el sema 'contable', por lo que exige que su término sea contable; en caso contrario, esta preposición se ve sustituida por la locución preposicional ANÁMESA SE: 
(37) a. Entre amigos.

b. Metaxý fílon.

(38) a. Entre Ana y Tomás.

b. Metaxý tis Ánnas kai tou Thomá.

(39) a. Está entre la arena.
b. Eínai *metaxý tis ámmou.

c. Eínai anámesa stin ámmo.

3.2.2.3. A: 'movimiento de proximidad sin extensión con contacto' / Se: 'movimiento de proximidad indiferente a la extensión con contacto e interioridad'

La preposición A expresa 'movimiento que establece contacto con el término y sus características', mientras que SE especifica que ese movimiento concluye 'en contacto y dentro del término’ (Tzártzanos 1991: 210); no obstante, a menudo esta diferencia resulta contextualmente irrelevante.

Como decimos, el movimiento de los verbos regentes dinámicos que se construyen con A concluye 'en contacto con el término y sus características'. Esto supone que cuando el término se caracteriza por la 'puntualidad' ('-extensión') el contacto se interpreta como 'contigüidad' (junto a); pero cuando el término posee 'extensión' o 'interioridad' (casa, país, etc.), el contacto de A se impregna de estas características, desarrollándose un sentido general de 'movimiento que concluye en el interior del término'. Por su parte, se intenta localizar el límite del movimiento no sólo en contacto, sino dentro del término; algo natural con términos que presentan 'extensión', pero imposible cuando el término designa algo puntual, lo que provoca una 'contigüidad' contextual similar a la de A:

(40) a. Voy a casa.

b. Pigaíno sto spíti.

(41) a. Corre a la puerta.

b. Tréchei stin pórta.

En entornos espaciales no dinámicos, temporales o nocionales, A neutraliza su 'movimiento', restándole los semas 'proximidad con contacto sin extensión' que, como hemos visto, constituyen una 'contigüidad' de la noción del regente con respecto al término y sus características (Trujillo Carreño 1971: 267-268; Morera Pérez 1988: 100-101, 159-160). En estos contextos, la preposición SE también neutraliza el 'movimiento', por lo que le quedan los semas 'proximidad con contacto, interioridad e indiferencia a la extensión', que dan paso al sema concomitante 'ubicación en el interior'. Ahora bien, cuando el término es puntual, la 'interioridad' es inviable, por lo que la 'ubicación en el interior' sólo puede interpretarse como 'contigüidad':

(42) a. Está a la mesa.

b. Eínai sto trapézi.

(43) a. Es a las tres.

b. Eínai stis treis.

El término de (42) está caracterizado por ‘+extensión', pero la 'puntualidad' de A impone un sentido de 'contigüidad' (junto a). No obstante, la 'ubicación en el interior' de se permite 
el desarrollo de un sentido tanto de 'ubicación' como de 'contigüidad' que depende de las características del sujeto (así, con una persona se interpretaría 'contigüidad', pero con un plato, 'ubicación'). El término de (43) es, sin embargo, puntual, por lo que tanto A como SE desarrollan 'contigüidad' (o 'puntualidad/exactitud'). No se debe considerar que los semas concomitantes 'puntualidad' de A y 'ubicación en el interior' de se sean sentidos contextuales, pues aparecen en todos los contextos en los que se neutraliza el 'movimiento', interactuando con el resto de los semas del contexto y desarrollando diversos sentidos contextuales.

Antes de concluir este apartado debemos mencionar que la remarcable frecuencia con que SE y A aparecen en contextos equivalentes, afecta incluso a casos en los que ambas preposiciones funcionan como índice gramatical, lo que es debido a que su principal diferencia, la 'interioridad' de SE, no tiene cabida en el mundo gramático-nocional:

(44) a. Le hablo a Nikos

b. Miló ston Níko.

3.2.2.4. En: 'ubicación con interioridad y extensión'/ Se: 'movimiento de proximidad indiferente a la extensión con contacto e interioridad'

Aunque SE y EN aparentemente no comparten semas, en realidad sí lo hacen, pues como acabamos de precisar, en entornos no dinámicos, SE desarrolla el sema concomitante 'ubicación en el interior', que es la propia definición componencial de EN:

(45) a. Está en casa.

b. Eínai sto spíti.

(46) a. En media hora llegaremos.

b. Se misí óra tha ftásoume.

(47) a. En mi opinión...

b. Sti gnómi mou..."

Recientemente hemos visto que a puede expresar una 'localización puntual o contigua', lo que le permite compartir ciertos contextos con EN; sin embargo, el uso de una u otra preposición conlleva en español una diferencia semántica que no se puede establecer en griego mediante SE sin recurrir a otros medios:

(48) a. Está al volante.

b. Eínai sto timóni ('Está en el volante'). / Eínai dípla sto timóni ('Está junto al volante’).

(49) a. Está a la mesa.

b. Eínai sto trapézi ('Está en la mesa'). / Káthetai sto trapézi ('Está sentado a la mesa').

(50) a. Está en el volante.

b. Eínai sto timóni ('Está en el volante'). / Eínai páno sto timóni ('Está sobre el volante').

(51) a. Está en la mesa.

b. Eínai sto trapézi ('Está en la mesa’). / Eínai páno sto trapézi ('Está encima de la mesa’).

11 La frecuencia de uso de la expresión katá ti gnómi mou, es mayor que la de sti gnómi mou, pero esta última es también habitualmente empleada. 
En (48)a y (49)a la 'contigüidad' de A expresa que algo 'está junto al término y expuesto a sus efectos o utilidad'; mientras que la 'ubicación entre límites' de eN en (50)a y (51)a indica que algo 'está dentro de los límites físicos o funcionales del término'. No obstante, en las traducciones propuestas en (48)b, (49)b, (50)b y (51)b se observa que la 'ubicación' de SE simplemente expresa una localización, por lo que se debe recurrir a otros medios para marcar los matices semánticos que conlleva el uso de A y EN.

Ahora bien, la realidad es que la definición componencial de se le permite aparecer en prácticamente todos los contextos no dinámicos que en español se reparten EN y A. De hecho, este uso locativo de SE es tan común, que el griego moderno no posee ninguna otra preposición que exprese exclusivamente esta noción.

\subsubsection{Preposiciones griegas y españolas sin semas comunes}

3.2.3.1. Ante: 'ubicación con referencia externa horizontal y positiva' / Bajo: 'ubicación con referencia externa horizontal y negativa' / Contra: 'movimiento de proximidad con bloqueo' / Sobre, 'ubicación con referencia externa vertical y positiva' / Tras: 'ubicación con referencia externa horizontal y negativa'

Estas preposiciones y sus definiciones componenciales son exclusivas del español, de modo que el griego debe echar mano de diversos recursos para representar los sentidos que desarrollan. Así, dado que ANTE, BAJO, CONTRA, SOBRE y TRAS tienen una precisión semántica similar a la de los adverbios nominales (Pavón 1999: 600-601, 605), en contextos interlingüísticamente equivalentes el griego suele recurrir a construcciones adverbiales ${ }^{12}$ con características nominales semejantes:

(52) a. Está ante /bajo/contra/sobre/tras la mesa.

b. Eínai brostá se, apó/káto apó/enántia se/páno se, apó/piso apó to kreváti.

\subsubsection{Por: 'movimiento con interioridad y extensión'}

POR no guarda ninguna relación con los adverbios nominales ni tiene referente externo, lo que hace que tenga un significado poco concreto y muy permeable al entorno, que desarrolle gran cantidad de sentidos contextuales y que la norma la haya dotado de gran cantidad de usos gramaticales. En griego no hay ninguna preposición con una composición semántica similar, de modo que cuando un grecófono desea expresar sentidos similares a los de POR, emplea recursos muy variados:

(53) a. No fuimos de vacaciones por la lluvia.

b. Den pígame diakopés exetías/lógo tis vrochís.

'No fuimos de vacaciones a causa/por razón de la lluvia'.

(54) a. Volamos a Atenas por Roma.

b. Petáxame stin Athína méso Rómis.

'Volamos a Atenas por medio de Roma'.

12 Entendemos que las locuciones preposicionales son construcciones adverbiales acompañadas de complemento. 
En español, POR es seleccionada frente a otros procedimientos más precisos cuando el contexto resulta claro o se pretende subrayar otros segmentos del mensaje. Pues bien, cuando los grecófonos quieren transmitir mensajes con estas mismas características, tienden a emplear APÓ y SE, preposiciones que, aunque tienen significados muy diferentes a POR, comparten con ella su carencia de referencia externa, su gran permeabilidad al contexto (lo que les permite expresar gran cantidad de sentidos) y los numerosos usos de los que la norma las ha cargado:

(55) a. Pasó por la ventana

b. Pérase apó to paráthiro.

(56) a. Fue diseñado por él.

b. Sxediástike apó ekeínon.

(57) a. Está por el parque.

b. Eínai sto parko.

Nos resultaría imposible hacer un recorrido por todos los sentidos contextuales de POR contrastando su correspondencia en griego. No obstante, para exponer nuestro planteamiento bastará con que nos centremos en la expresión del 'tránsito' (55), la 'agentividad' (56) y la 'indeterminación de movimiento y de ubicación' (57). Tres usos especialmente característicos de POR que permiten explicar muchos de los significados que habitualmente se le atribuyen, y las razones que motivan que en griego se recurra a unos u otros medios para expresar esos mismos sentidos.

\section{a. 'Tránsito'}

La expresión del 'tránsito' vista en (55) es el significado más propio de POR, ya que el 'movimiento con extensión e interioridad' desarrolla de manera natural el sema concomitante 'tránsito'.

La preposición que en griego expresa habitualmente 'tránsito' es APÓ, pero por razones semánticas muy diferentes. El 'movimiento de alejamiento' de esta preposición es indiferente a la 'extensión', de manera que no expresa el 'origen absoluto del movimiento' (como hace DESDE), sino el 'punto desde el que se considera el movimiento'. Ello, sumado al hecho de que los verbos de desplazamiento (no así los verbos carentes de 'desplazamiento' como PERPATÓ, 'caminar') presuponen un complemento, explícito o no, que expresa el 'punto final' de la acción verbal, posibilita que el término sea interpretado como 'espacio que debe ser atravesado para llegar a un lugar', desarrollándose así un sentido de ‘tránsito’:

(58) a. La luz entra por una ventana (en la habitación).

b. To fos baínei apó éna paráthiro (sto domátio).

(59) a. Los caracoles salieron (fuera) por un agujero de la caja.

b. Ta saligkária vgíkane (éxo) apó mía trípa tis koútas.

(60) a. Los niños van al parque por la entrada principal.

b. Ta paidiá páne sto párko apó tin kentrikí pýli.

(61) a. Los fantasmas pasan (a algún sitio) por las paredes.

b. Ta fantásmata pernáne (mésa) apó tous toíchous.

(62) a. Lo arrastró por la puerta.

b. Ton ésyre apó tin pórta (kápou). 
(63) a. Trajo la madera (aquí) por la ventana.

b. Éfere ta xýla (edó) apó to paráthiro.

Y, por las mismas razones, en contextos carentes de movimiento, el 'tránsito' de POR desarrolla un sentido de 'medio a través del que se realiza la acción verbal' (Morera Pérez 1988: 314).

\section{b. 'Agentividad'}

La expresión del complemento agente es una función sintáctico-semántica codificada en griego mediante APÓ y en español mediante POR, pero por distintas causas.

Hemos visto que el 'tránsito' de POR expresa que el término es el 'medio a través del que se realiza la acción verbal'. Sin embargo, las acciones pasivas son sufridas, no realizadas, por el sujeto, por lo que el término se transforma en el 'medio a través del que se sufre la acción verbal'. Partiendo de esta base, se pueden atribuir distintas funciones al grupo preposicional con POR. Así, se suele considerar que con sujetos inanimados este grupo preposicional se codifica como expresión de la 'causa' o el 'medio', pero como 'agente' de la acción verbal con sujetos animados:

(64) Fue ofendido por Nikos.

(65) Fue ofendido por las palabras de Nikos.

'agentividad'

'causa'

Sin embargo, esta diferenciación es puramente normativa; de hecho, como se manifiesta en (65), no siempre es posible diferenciar estas interpretaciones. Ésta es la razón por la que los complementos de causa introducidos mediante POR en las oraciones pasivas, a menudo adquieren la función de sujeto cuando estas oraciones son transformadas en activas, (66). Y lo mismo sucede cuando se reestructura el complemento de causa de una oración no pasiva, (67):

(66) Las palabras de Nikos le ofendieron.

(67) a. Me he asustado por el ruido.

b. El ruido me ha asustado.

Por su parte, en estos mismos contextos el 'alejamiento' de APÓ interpreta al término como el 'origen' de la acción verbal ${ }^{13}$. A partir de este punto todo sigue los mismos cauces que hemos visto en español, ya que con términos animados el grupo preposicional suele ser considerado complemento agente, y con inanimados, complemento de causa, medio, instrumento, etc.:

(68) Prosvlíthike apó ta lógia tou Níkou.

'Fue ofendido por las palabras de Nikos'

'causa'

13 En español actual la preposición DE como introductora de complemento agente está restringida a verbos de conocimiento, afecto, compañía y estimación; no obstante, hasta el siglo XVII era habitualmente empleada para introducir este complemento. Ello obedece a las mismas razones semánticas que permiten que APó introduzca el agente en griego, pues el 'origen sin extensión' de DE significa la relación como 'origen'; ámbito vedado a DESDE porque su ‘+extensión' no encaja en entornos nocionales. 
(69) Prosvlíthike apó ton Níko. 'Fue ofendido por Nikos'

'agente'

Sin embargo, al igual que en español, esto no es sino una decisión normativa, ya que APÓ expresa el 'origen' en todos los casos. Por esa misma razón, no siempre es fácil diferenciar la agentividad de otras nociones, como sucede en (68). Y tanto los complementos agente como los casuales, se transforman en sujetos cuando se reestructura la oración, (70), (71) y (72):

(70) Prosvlíthike apó ton Níko. $\rightarrow$ O Níkos ton prosévale.

'Fue ofendido por Nikos'. $\rightarrow$ 'Nikos lo ofendió'.

(71) Prosvlíthike apó ta lógia tou Níkou. $\rightarrow$ Ta lógia tou Nikou ton prosévalan.

'Fue ofendido por las palabras de Nikos'. $\rightarrow$ 'Las palabras de Nikos lo ofendieron'.

(72) Trómaxa apó ton thórivo. $\rightarrow \mathrm{O}$ thórivos me trómaxe.

'Me asusté por el ruido'. $\rightarrow$ 'El ruido me asustó'.

c. 'Indeterminación de movimiento y de ubicación'

En contextos estáticos o dinámicos que no implican 'desplazamiento', POR neutraliza su 'movimiento', restándole únicamente los semas 'extensión con interioridad', los cuales sitúan la noción del verbo en alguno de los puntos dentro de los límites impuestos por el término, desarrollándose así un sentido de 'indeterminación', interpretable como 'en cualquier lugar del término’ (Morera Pérez 1988: 291):

(73) Hay muchas estatuas por la plaza.

(74) Mi abuelo camina por el parque.

(75) El león daba vueltas por la jaula.

En estos contextos SE también neutraliza su 'movimiento', pasando a expresar 'ubicación absoluta' y situando la noción del verbo dentro de los límites del término, pero sin añadir la 'indeterminación' de POR. No obstante, este matiz puede surgir también de la propia referencia del término, del mismo modo que surgiría en español si en los ejemplos indicados se empleara EN en lugar de POR:

(76) Échei pollá agálmata stin plateía.

'Hay muchas estatuas en la plaza'.

(77) O pappoús mou perpatáei sto párko.

'Mi abuelo camina en el parque'.

(78) To liontári ékane kýklous sto kelí.

'El león daba vueltas en la jaula'.

Aunque hemos quedado lejos de analizar todos los usos de POR y sus correspondencias en griego, los sentidos seleccionados ('tránsito', 'agentividad' e 'indeterminación') sientan las bases para explicar tanto el modo en que surgen el resto de los sentidos contextuales como las razones que justifican que en griego se recurra a unos u otros medios para expresar estos mismos sentidos. 


\subsubsection{Pará: ‘contraposición’ / Antí: ‘contraposición con exclusión' ('sustitución')}

PARÁ y ANTí no se corresponden con ninguna preposición española y tienen significados muy concretos (Poulopoúlou 1999: 366-377), por esa razón en español se representan sus sentidos contextuales (habitualmente muy semejantes a sus significados) recurriendo principalmente a construcciones adverbiales:

(79) a. Pará ta ósa eípane, den írthane.

b. A pesar de lo que dijeron, no vinieron.

(80) a. Pará ti thélisí tou.

b. En contra de/contra su voluntad.

(81) a. Antí tou Giánni, írthe o Níkos.

b. En lugar de Gianni, vino Nikos.

(82) a. Tha milísei i Évi antí tou Micháli.

b. Hablará Evi en lugar de Michalis.

\section{CONCLUSIONES}

Creemos que con el presente artículo se ha demostrado que el análisis componencial es adecuado para contrastar los paradigmas preposicionales de distintas lenguas, ya que este método considera que las preposiciones tienen un único significado; toma en consideración exclusivamente factores lingüísticos, no referentes extralingüísticos; y contrasta elementos lingüísticos de la misma lengua o de lenguas diferentes mediante la comparación de sus semas.

También ha quedado demostrado que, aunque la norma de una lengua altera la frecuencia y los campos de uso de las palabras, las preposiciones griegas y españolas que presentan mayor similitud semántica tienen más posibilidades de aparecer en entornos interlingüísticamente semejantes.

En este sentido, se ha destacado que las preposiciones griegas y españolas están caracterizadas por un total de veinte semas, siendo catorce de ellos comunes a ambos paradigmas preposicionales. Esto posibilita que algunos de ellos sean compartidos por preposiciones del griego y del español, y que incluso haya preposiciones de ambas lenguas que presenten exactamente la misma definición componencial. Asimismo, este reducido número de semas limita necesariamente sus combinaciones, lo que permite que algunas preposiciones que aparentemente no tienen correspondencia semántica en la otra lengua, desarrollen semas concomitantes similares a los significados de las preposiciones de la otra lengua.

Así, salvo que la norma lo impida, es de esperar que las preposiciones que tienen combinaciones semánticas idénticas (HASTA/MÉCHRI, CON/ME, SIN/CHORÍS, PARA /GIA, HACIA/PROS y SEGÚN/KATÁ), aparezcan en los mismos contextos y desarrollen sentidos muy similares.

Por la misma razón, las preposiciones que presentan algunos semas comunes (DE-DESDE/ APÓ, ENTRE/METAXÝ, A/SE y EN/SE) son susceptibles de compartir, interlingüísticamente hablando, aquellos contextos en los que sus semas comunes están activos.

$\mathrm{Y}$, al contrario, los sentidos que desarrollan las preposiciones que no tienen correspondencia semántica con preposiciones de la otra lengua, suelen expresar estos mismos sentidos mediante otro tipo de recursos. En el caso de las preposiciones que tienen significados muy concretos (ANTE, BAJO, CONTRA, SOBRE y TRAS en español; PARÁ y ANTí, en griego), estos 
recursos suelen consistir en expresiones adverbiales. Muy diferente es el caso de POR, cuyos sentidos suelen ser expresados en griego mediante APÓ y SE, preposiciones que cuentan con una imprecisión semántica y una amplitud de campos de usos equiparables a los de APÓ.

Con este estudio ponemos de manifiesto las relaciones semánticas que se dan entre las preposiciones griegas y españolas, algo que se justifica por sí mismo en el ámbito del contraste interlingüístico, pues es un paso más a la hora de esclarecer las relaciones estructurales entre el griego y el español. No obstante, también tiene una aplicación directa para la docencia de estas lenguas a hablantes no nativos, pues las preposiciones presentan un alto grado de dificultad (Leontaridi y Pérez 2008; Leontaridi y Peramos, 2011) debido a la multiplicidad de significados, a menudo inconexos, que se les atribuyen. Por esa razón, consideramos que el conocimiento profundo de las características semánticas de las preposiciones, con independencia de los contextos en los que éstas aparezcan, capacitará al docente para transmitir a sus alumnos las claves que les permitan interiorizar el funcionamiento y correcto empleo de estos elementos.

\section{Referencias bibliográficas}

Alarcos Llorach, E. (2001). Gramática de la lengua española. Madrid: Espasa.

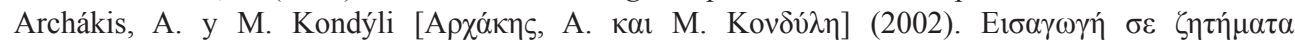

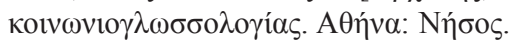

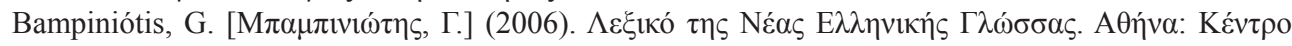
$\Lambda \varepsilon \xi$ iкодоүías.

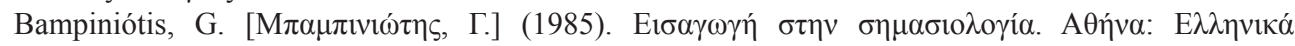
Гро́ $\mu \mu \alpha \tau$.

Bádenas de la Peña, P. (1988). "La situación lingüística en Grecia. Problemas y perspectivas”, Erytheia. Revista de estudios bizantinos y neogriegos, año 9, $\mathrm{n}^{\circ}$ 2, pp. 303-333.

Bortone, P. (2010). Greek prepositions. From antiquity to the present. Oxford: Oxford University Press.

Bosque, I. y V. Demonte (dirs.) (1999). Gramática descriptiva de la lengua española. Madrid: Espasa.

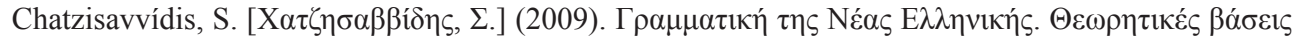

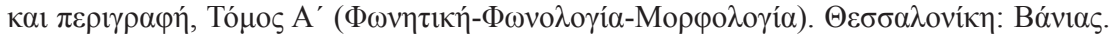

Cifuentes Honrubia, J. L. (1998). "Semántica y cognición de los usos prepositivos en español”. En Ramón Trives, E. y E. Provencio Garrigós (coords.). Estudios de Lingüistica Textual. Homenaje al Profesor M. Muñoz Cortés:XVIII Curso de Lingüistica Textual. Murcia: Universidad de Murcia, pp. 107-121.

De Bruyne, J. (1999). "Las preposiciones”. En I. Bosque y V. Demonte (dirs.), pp. 657-703.

Fykias, I. (1997). "The case system in Modern Greek: evidence from PPs". En Gaberell Drachman et al. (eds.). Proceedings of the 2nd International Conference on Greek Linguistics. Vol. 2. Graz: W. Neugebauer Verlag GmbH, pp. 439-448.

Gómez Laguna, I. (2014): Análisis contrastivo de la estructura semántica de las preposiciones en español y griego. Usos contrastivos de la preposición A. Tesis doctoral inédita, Universidad Aristóteles de Salónica.

Goodenough, W. H. (1956). "Componential analysis and the study of meaning”, Language, año 32, $\mathrm{n}^{\mathrm{o}} 1$, pp. 195-216.

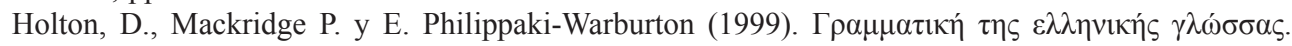

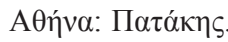

Horno Chéliz, Ma . C. (2002). Lo que la preposición esconde. Zaragoza: Prensas universitarias de Zaragoza. 
Katz, J. J. y J. A. Fodor (1963). “The structure of a semantic theory", Language, año 39, $\mathrm{n}^{\mathrm{o}}$ 2, pp. 170-210.

Kempson, R. M. (1977). Semantic theory. Cambridge: Cambridge University Press.

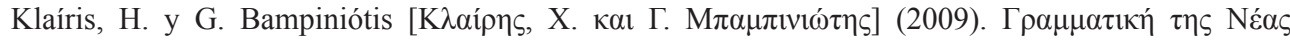

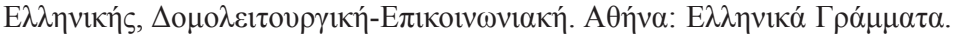

Laguna Campos, J. (2005). "Gramática de las preposiciones". En Castillo Carballo, Mª A., Cruz Moya, O., García Platero, J. M. y Moya Gutiérrez, J. P. (coords.). Actas del XV Congreso Internacional de ASELE. Sevilla: Universidad de Sevilla, pp. 526-533.

Lamíquiz Ibáñez, V. (2001). Lengua española: método y estructuras lingüisticas. Barcelona: Ariel.

Leech, G. (1977). Semántica. Madrid: Alianza.

Leontaridi, E. (2011). "Paralelos (?) de temporalidad en español y en griego: el caso de los tiempos del pasado del indicativo". En Sinner, C., E. Hernández Socas y Bahr Ch. (eds.). Tiempo, espacio y relaciones espacio-temporales. Nuevas aportaciones de los estudios contrastivos. Frankfurt am Main: Peter Lang.

Leontaridi, E. (2008 [2001]). Los tiempos del pasado del indicativo en español y en griego. Madrid: Biblioteca virtual redELE, 9 ( $2^{\circ}$ semestre 2008). Tesis doctoral, Universidad de Salamanca: http:// www.mecd.gob.es/dctm/redele/Material-RedEle/Biblioteca/2008_BV_09/2008_BV_09_2_semestre/2008_BV_09_15Leontaridi.pdf?documentId=0901e72b80e2ad5e (25-11-2017).

Leontaridi, E. y Peramos, N. (2011). "El uso del paradigma preposicional español por grecófonos desde el punto de vista morfológico, sintáctico y semántico". En Ridruejo, E. y Mendizábal, N. (eds.). Actas del IX Congreso Internacional de Lingüistica General. Valladolid: Universidad de Valladolid, 1338-1356. <https://goo.gl/UpA6bB >

Leontaridi, E. y Pérez, R.M. (2008). Claves del español para hablantes de griego. Madrid: SM.

Lyons, J. (1997). Semántica lingüística. Una introducción. Barcelona: Paidós.

Marcos Marín, F. A. y P. España Ramírez (2001). Guía de gramática de la lengua española. Madrid: Espasa.

Morera Pérez, M. (1988). Estructura semántica del sistema preposicional del español moderno y sus campos de usos. Puerto del Rosario: Cabildo de Fuerteventura.

Morera Pérez, M. (1999). Apuntes para una gramática del español de base semántica. Puerto del Rosario: Cabildo de Fuerteventura.

Osuna García, F. (1991). Función semántica y función sintáctica de las preposiciones. Málaga: Librería Ágora.

Osuna García, F. (2008). "Las funciones semánticas de los morfemas auxiliares". En Actas del 37 Simposio Internacional de la Sociedad Española de Lingüística (SEL). Pamplona: Universidad de Navarra, pp. 625-637.

Papanastassiou, G. (2010). "Katharevousa: Its nature and contribution to Modern Greek". En Greek, a language in evolution. Essays in honour of Antonios N. Jannaris. Hildesheim, Zurich, New York: Georg Olms Verlag, pp. 227-267.

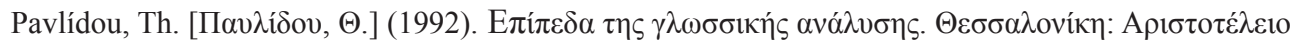

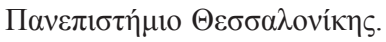

Pavón Lucero, Ma . V. (1999). "Clases de partículas: preposición, conjunción y adverbio". En Bosque de, I. y V. Demonte (dirs.), pp. 565-655.

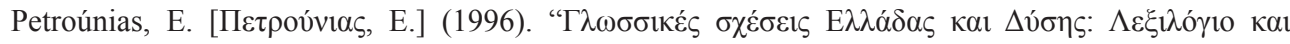

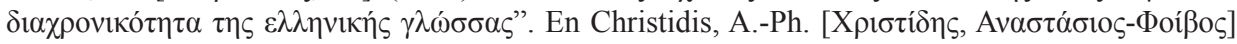

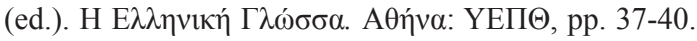

Portilla Chaves, M. (2011). "El origen de las preposiciones en español", Revista de Filología y Lingüistica de la Universidad de Costa Rica, año 37, nº 1, pp. 229-244.

Pottier, B. (1964). "La semántica y los criterios funcionales". En Actas del Primer Congreso Internacional de Hispanistas en Oxford. Oxford: The dolphin book, pp. 415-420.

Pottier, B. (1970). Lingüistica moderna y filología hispánica. Madrid: Gredos. 


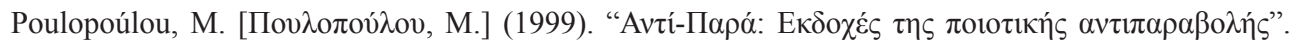

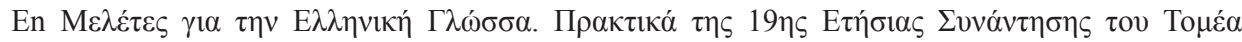
Г

RAE (2009) = Real Academia Española. Nueva gramática de la lengua española. Morfología y sintaxis. Madrid: Espasa.

RAE (2001) = Real Academia Española. Diccionario de la Lengua Española, 22a Edición. Madrid: Espasa.

Rodríguez Adrados, F. (1999). Historia de la lengua griega. Madrid: Gredos.

Rodríguez Zamora, José M. (2004). "Análisis estructural y significado lingüístico”, Revista de Filología y Lingüistica de la Universidad de Costa Rica, año 30, $\mathrm{n}^{\circ}$ 1, pp. 181-203.

Seco Reymundo, M. (1996). Gramática esencial del español. Madrid: Espasa.

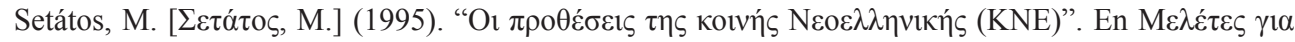

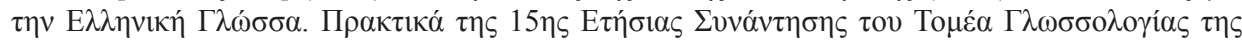

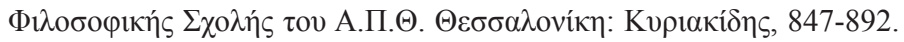

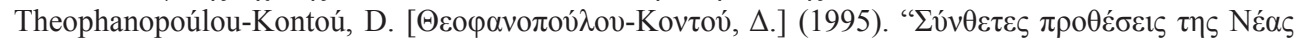

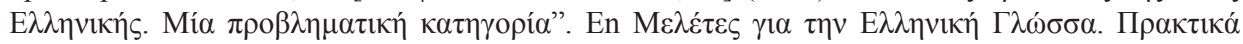

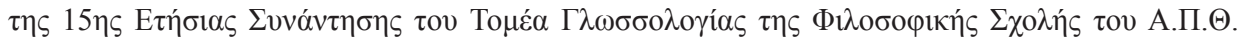

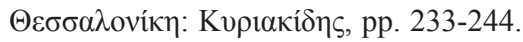

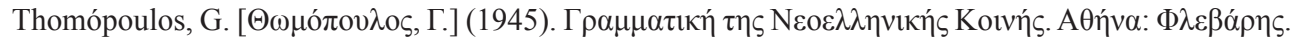
Trujillo Carreño, R. (1971). "Notas para un estudio de las preposiciones españolas". Thesaurus: Boletín del Instituto Caro y Cuervo, año 26, nº 2, pp. 234-279.

Trujillo Carreño, R. (1988). Introducción a la semántica española. Madrid: Arco.

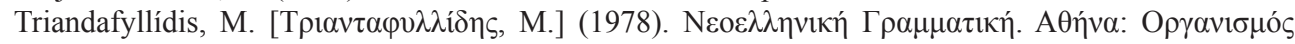

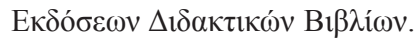

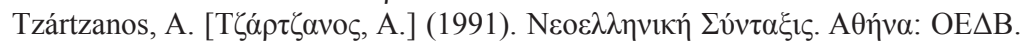


